\title{
A Remark on the Energy Transfer in Biological Systems
}

Avery, Bay \& Szent-Györgyi (196r) considered in their paper two mechanisms of energy transfer in biological systems: an individual and a collective one. The individual picture concerned an aggregate of molecules in which an energy quantum is deposited; this quantum excites a molecule and the excitation is transported from one molecule to another by random jumps until it reaches a sink in the aggregate. The authors stated that the individual picture would only be valid if $\frac{\Gamma \mathrm{t}_{1}}{\mathrm{n}^{2}}>\mathrm{I}$, where $\hbar \Gamma$ is the width of the excited level, $t_{1}$ is the time needed for the excitation to jump to an adjacent molecule and $n$ the number of paths an excitation can use to jump to a next nearest neighbour molecule. $\nmid$ The authors assumed that $\Gamma$ would be of the order of ${ }_{10} 0^{13} \mathrm{sec}^{-1}$ and calculated $t_{1}$ using the number of random jumps which the excitation would need to reach the sink. They concluded that the individual picture cannot be valid in a system of 200 chlorophyll molecules. We have carried out the calculation in a slightly different way using a more accurate estimate of the number of random jumps and have arrived at the conclusion that it is not possible to give a definite answer as to the validity of the individual picture.

Let us consider, as Avery et al. (1961) did, a monomolecular layer. Let us, to simplify our calculation, assume that it is a square layer of $\mathrm{N}^{2}$ molecules and that the sink is in the centre of the square. To a good approximation this picture is equivalent to a square with a sink in each corner. Let us now take a coordinate system as illustrated (Fig. I). We call $D_{x y}$ the number of jumps to be taken at random to reach the sink from $(x, y)$. Only single jumps, parallel to one of the axes and to the nearest neighbour, are allowed. The boundary conditions are now:

and

$$
D_{0, y}=D_{N, y} \text { for all } y
$$

It is clear that

$$
D_{x, 0}=D_{x, N} \text { for all } x
$$

$$
\mathrm{D}_{0,0}=0
$$

Further it is clear that the number of jumps from $(x, y)$ equals the average

$\dagger$ Our definition for $n$ is equivalent to the definition used by Avery et al.: $n$ is the number of intermediate neighbour molecules, which can be involved in a jump to a definite next nearest neighbour molecule. 
of the numbers for the nearest neighbours of $(x, y)$ plus one jump. This gives the recurrence relation:

$$
D_{x, y}=\frac{1}{4}\left(D_{x+1, y}+D_{x-1, y}+D_{x, y+1}+D_{x, y-1}\right)+I
$$

for all $D_{x, y}$ except $D_{0,0}$.

We now consider the average $D$ of all $D_{x, y}$ over the aggregate:

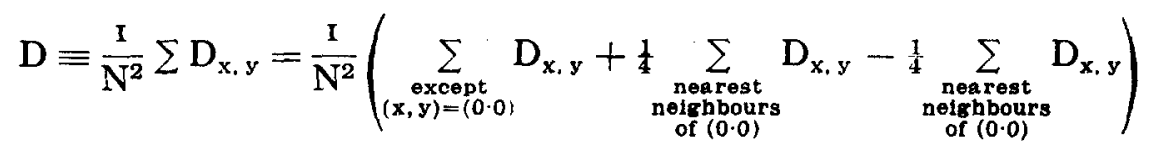

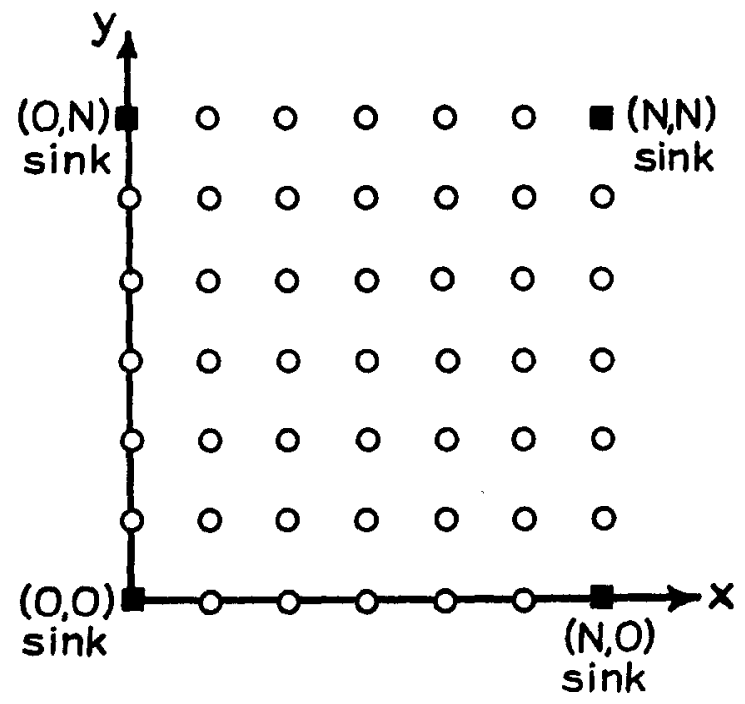

Frc. I. Coordinate system for a square layer of $\mathrm{N}^{2}$ molecules with a sink in each corner.

Substituting equation (4) in the first term we find for the sum of the first and second term $\frac{N^{2} D+N^{2}-I}{N^{2}}$. As the third term equals $\frac{D_{1,0}}{N^{2}}$ we find:

So

$$
\mathrm{D}=\mathrm{D}+\frac{\mathrm{N}^{2}-\mathrm{I}}{\mathrm{N}^{2}}-\frac{\mathrm{D}_{1.0}}{\mathrm{~N}^{2}}
$$

$$
D_{1,0}=N^{2}-I
$$

The general solution of $D_{x, y}$ is then:

$$
D_{x, y}=-\left(x^{2}+y^{2}\right)+a(x+y)+b x y+c
$$

Combining equations ( 1 ), (2), (5) and (6) we find

$$
D_{x, y}=-\left(x^{2}+y^{2}\right)+N(x+y)+N^{2}-N
$$


for all $(x, y)$ except $(0,0)$ and

This gives

$$
\mathrm{D}_{0.0}=0
$$

$$
D \equiv \frac{I}{N^{2}} \sum_{x, y} D_{x, y} \approx \frac{I}{N^{2}} \int_{0}^{N} \int_{0}^{N} d x d y D_{x, y}=\frac{4}{3} N^{2}-N
$$

The number of jumps to be taken is thus of the order of the number of molecules in the aggregate. Avery $e t$ al. stated that the number of jumps should be of the order of (number of molecules) ${ }^{2}$.

Let us now follow their calculation of $t_{1}$ in a system of 200 chlorophyll molecules. More recent values than those employed by Avery $e$ t al. (196r) for the fluorescence yield of chlorophyll in vivo given by Latimer, Bannister \& Rabinowitch (1957) indicate a yield of about $2 \times 10^{-2}$. The number of jumps is according to our formula about $25^{\circ}$, so $t_{1}$ should be about $8 \times 10^{-13} \mathrm{sec}$ and $\frac{\Gamma \mathrm{t}_{1}}{\mathrm{n}^{2}} \approx 2$.

This would lead to the conclusion that the individual picture can be valid, in contradiction with the conclusion of Avery et al. (I96r). However, the condition $\frac{\Gamma \mathrm{t}_{1}}{\mathrm{n}^{2}}>\mathrm{I}$ for the validity of the individual picture is a necessary, but not a sufficient one. Therefore it is not possible to give a definite answer about the validity of the individual picture. Both the mechanism which causes the excitation to jump and the probability of jumps longer than a single one should be studied before a sufficient condition can be formulated.

Physical Laboratory $\&$ Institute for Theoretical Physics,

State University, Utrecht, Netherlands.

(Received 19 October 1962)

\section{REFERENCES}

Avfry, J., Bay, Z. \& Szent-Györgyi, A. (196r). Proc. nat. Acad. Sci., Wash. 47, 742. Latimer, P., Bannister, T. T. \& Rabinowitch, E. I. (1957). In "Research in Photosynthesis," pp. 107-112. Interscience, New York. 